Pacific Journal of Mathematics

MOMENT SEQUENCES OBTAINED FROM RESTRICTED 


\title{
MOMENT SEQUENCES OBTAINED FROM RESTRICTED POWERS
}

\author{
RODNEY NILLSEN
}

Let $\left(m_{n}\right)_{n=1}^{\infty}$ be an increasing divergent sequence of positive numbers. Then we are interested in characterising those sequences $\left(\alpha_{n}\right)_{n=1}^{\infty}$ for which $\alpha_{n}=\int_{0}^{1} x^{m_{n}} f(x) d x$ for $n=1,2, \cdots$ and some $f \in L^{2}([0,1))$. It is shown that if $\left(m_{n}\right)_{n=1}^{\infty}$ diverges sufficiently rapidly, then $\sum_{n-1}^{\infty}\left|\alpha_{n}\right|^{2}<\infty$ if and only if $\alpha_{n}=\sqrt{m_{n}} \int_{0}^{1} x^{m_{n}} f(x) d x$ for $n=1,2, \cdots$ and some $f \in L^{2}([0,1))$. It is also shown that if $\left(m_{n}\right)_{n=1}^{\infty}$ is a lacunary sequence of integers then the Hilbert subspace of $L^{2}([0,1))$ generated by the functions $x^{m}(n=1,2, \cdots)$ has a reproducing kernel.

1. Introduction. Let $C([0,1])$ denote all the complex valued continuous functions on $[0,1]$. If $\alpha \geqq 0$ let $e_{\alpha}$ be the function in $C([0,1])$ given by $x \rightarrow x^{\alpha}$. Throughout the paper, $S$ will be a given sequence $\left(m_{n}\right)_{n=1}^{\infty}$ of positive real numbers so that $0 \leqq m_{1}<m_{2}<$ $m_{3}<\cdots$ and $\lim _{n \rightarrow \infty} m_{n}=\infty$. The subspace of $C([0,1])$ obtained by taking the uniform closure of the vector space generated by $\left\{e_{m_{i}}: i=1,2, \cdots\right\}$ will be denoted by $M(S)$.

A classical result due to Müntz and Szasz (see, for example, [2] p. 272) says that if $m_{1}=0, M(S)=C([0,1])$ if and only if $\sum_{i=2}^{\infty} 1 / m_{i}=\infty$. In the case where $\sum_{i=2}^{\infty} 1 / m_{i}<\infty$ it can be shown that $e_{\alpha}$ is not in $M(S)$ unless $\alpha=m_{i}$ for some $i$ ([7], p. 305). It follows from this, using the Hahn-Banach theorem, that if $\sum_{i=2}^{\infty} 1 / m_{i}<\infty$ and if $j$ is given, then there is a measure $\mu$ so that $\int_{0}^{1} x^{m_{i}} d \mu(x)=0$ if $i \neq j$ and $\int_{0}^{1} x^{m_{j}} d \mu(x) \neq 0$. Among the results of this paper, it is shown that if $S$ satisfies a certain stronger condition than $\sum_{i=2}^{\infty} 1 / m_{i}<\infty$, the measure $\mu$ can be chosen to be absolutely continuous with respect to Lebesgue measure and at the same time be supported by $[0, \delta]$, where $\delta>0$ is preassigned.

We also let $L^{2}$ be the Hilbert space of all square integrable functions on $[0,1)$ and we denote by $A(S)$ the subspace of $L^{2}$ obtained by taking the closure, in the $L^{2}$ norm, of the vector space generated by $\left\{e_{m_{n}}: n=1,2, \cdots\right\}$. Any function in $M(S)$, if restricted to $[0,1)$, belongs to $A(S) . \quad S=\left(m_{n}\right)_{n=1}^{\infty}$ is said to be lacunary (or a Hadamard set) if there is $\alpha>1$ so that $m_{n+1}>\alpha m_{n}$, for $n=1,2,3, \cdots$. Lacunary sets are well known in complex analysis ([8], pp. 314-316) and in harmonic analysis ([7], pp. 100-118). We show that if $S$ is lacunary then $A(S)$ has a reproducing kernel.

Finally we shall be concerned with characterizing those sequences 
$\left(\alpha_{n}\right)_{n=1}^{\infty}$ which are of the form $\alpha_{n}=\int_{0}^{1} f(x) x^{m_{n}} d x$ for $n=1,2, \cdots$, for some $f \in L^{2}$. As noted in [3], vol. II, pp. 139-140, any such sequence $\left(\alpha_{n}\right)_{n=1}^{\infty}$ belongs to $\ell^{2}$, the Hilbert space of all square summable sequences on the positive integers. It is shown that, regardless of what $S$ is, it is never possible to obtain all of $\ell^{2}$ simply by taking different functions $f$ in $L^{2}$. However it is possible to obtain all of $\ell^{2}$ in this way if we consider instead sequences $\left(\alpha_{n}\right)_{n=1}^{\infty}$ of the form $\alpha_{n}=\sqrt{m_{n}} \int_{0}^{1} x^{m_{n}} f(x) d x$, provided the sequence $S$ diverges rapidly enough.

The basic idea underlying a number of our results is that provided there are "sufficiently large" gaps between $m_{i}$ and $m_{i+1}$ for $i=1,2, \cdots$, then the functions $\left\{e_{m_{i}}: i=1,2, \cdots\right\}$ are "sufficiently orthogonal" for them to be treated (in a certain sense) as orthogonal functions.

2. Properties of $A(S)$. In $L^{2}$ the Gram-Schmidt process can be applied to the functions $e_{m_{1}}, e_{m_{2}}, \cdots$ to obtain an orthonormal sequence $p_{1}, p_{2}, \cdots$ Of course we can write $p_{n}=\sum_{j=1}^{n} a_{n j} e_{m_{j}}$ or

$$
p_{n}(x)=\sum_{j=1}^{n} a_{n j} x^{m_{j}}, \quad \text { for } \quad 0 \leqq x<1 \text { and } n=1,2,3, \cdots \text {. }
$$

If the inner product in $L^{2}(0,1)$ is denoted by $(\cdot, \cdot)$ then also we have

$$
\left(p_{n}, p_{m}\right)=0 \quad \text { if } m \neq n \text {, and } 1 \text {, if } m=n .
$$

Then functions in $A(S)$ are precisely those functions of the form $\sum_{n=1}^{\infty} \alpha_{n} p_{n}$ for some sequence $\left(\alpha_{n}\right)_{n=1}^{\infty}$ such that $\sum_{n=1}^{\infty}\left|\alpha_{n}\right|^{2}<\infty$, where the series is to be interpreted in terms of the $L^{2}$ norm. We shall assume that the Gram-Schmidt process has been carried out so that $a_{n n}>0$ for all $n$, in which case the constants $a_{n j}$ in (2.1) are uniquely determined. If $S$ is a sequence of integers, it should be noted that the $p_{n}$ are polynomials and can be regarded as being defined on $D=\{\lambda:|\lambda|<1\}$.

LEMMA 2.1 .

$$
a_{11}=\sqrt{2 m_{1}+1} \quad \text { and } \quad a_{21}=-\sqrt{2 m_{2}+1}\left[\frac{2 m_{1}+1}{m_{2}-m_{1}}\right] .
$$

If $n>2$ and $j<n$ we have

$$
a_{n j}=(-1)^{n+j} \sqrt{2 m_{n}+1}\left[\frac{2 m_{j}+1}{m_{n}-m_{j}}\right]_{\substack{i=1 \\ i \neq j}}^{n-1}\left[\frac{m_{i}+m_{j}+1}{\left|m_{i}-m_{j}\right|}\right] .
$$

If $n>1$ we have 


$$
a_{n n}=\sqrt{2 m_{n}+1} \prod_{i=1}^{n-1}\left[\frac{m_{n}+m_{i}+1}{m_{n}-m_{i}}\right] \text {. }
$$

Proof. If $f_{1}, f_{2}, \cdots, f_{n} \in L^{2}([0,1))$, let $g\left(f_{1}, f_{2}, \cdots, f_{n}\right)$ be the determinant of $\left(\left(f_{i}, f_{j}\right)\right)_{1 \leq i, j \leq n}$ (the Gramian determinant, see [2], p. 177). Then by [2], p. 183 we have

$$
p_{n}=\frac{1}{\sqrt{g\left(e_{m_{1}}, e_{m_{2}}, \cdots, e_{m_{n}}\right) g\left(e_{m_{1}}, e_{m_{2}}, \cdots, e_{m_{n-1}}\right)}}\left|\begin{array}{ccc}
\left(e_{m_{1}}, e_{m_{1}}\right) & \cdots & \left(e_{m_{n}}, e_{m_{1}}\right) \\
\vdots & & \vdots \\
\left(e_{m_{1}}, e_{m_{n-1}}\right) & \cdots & \left(e_{m_{n}}, e_{m_{n-1}}\right) \\
e_{m_{1}} & \cdots & e_{m_{n}}
\end{array}\right| \text {. }
$$

Since $\left(e_{m_{i}}, e_{m_{j}}\right)=1 /\left(m_{i}+m_{j}+1\right)$, it is possible to use (2.6) to find the $a_{n j}$ and we find that (2.3), (2.4), and (2.5) are true. The calculation is tedious but straight forward and is similar to one used in one proof of the Müntz-Szasz theorem (see [2], pp. 270-271).

LEMma 2.2. $S$ is lacunary if and only if there is a number $C$ so that for all $n \geqq 2$,

$$
\left|\prod_{\substack{i=1 \\ i \neq j}}^{n}\left(\frac{m_{i}+m_{j}+1}{m_{i}-m_{j}}\right)\right| \leqq C, \quad \text { when } j \leqq n .
$$

Proof. Each term of the product in (2.7) is greater than 1, so that if (2.7) holds, $C>1$. In this case, for $n>1$ we will have $\left(m_{n}+m_{n-1}+1\right) \leqq C\left(m_{n}-m_{n-1}\right) \quad$ so that $m_{n} \geqq(C+1) /(C-1) m_{n-1}$, and $S$ must be lacunary.

Conversely, if $S$ is lacunary and $m_{1}>0$, choose $\alpha>1$ so that $m_{i+1}>\alpha m_{i}$ for $i=1,2, \cdots$. Then if $i>j$ we have

$$
m_{i}>\alpha^{i-j} m_{j} \text {. }
$$

If $x \geqq 0$ then $1+x \leqq e^{x}$ so that if $j \leqq n-1$,

$$
\prod_{i=j+1}^{n}\left(\frac{m_{i}+m_{j}+1}{m_{i}-m_{j}}\right)=\prod_{i=j+1}^{n}\left(1+\frac{2 m_{j}+1}{m_{i}-m_{j}}\right) \leqq e^{s j, n},
$$

where

$$
s_{j, n}=\sum_{i=j+1}^{n}\left(\frac{2 m_{j}+1}{m_{i}-m_{j}}\right) .
$$

If $j>1$, we also have

$$
\prod_{i=1}^{j-1}\left(\frac{m_{i}+m_{j}+1}{m_{j}-m_{i}}\right)=\prod_{i=1}^{j-1}\left(1+\frac{2 m_{i}+1}{m_{j}-m_{i}}\right) \leqq e^{t_{j}},
$$


where

$$
t_{j}=\sum_{i=1}^{j-1}\left(\frac{2 m_{i}+1}{m_{j}-m_{i}}\right) .
$$

Now

$$
\begin{aligned}
s_{j, n} & =2 \sum_{i=j+1}^{n}\left(\frac{1}{m_{i} / m_{j}-1}\right)+\sum_{i=j+1}^{n}\left(\frac{1}{m_{i}-m_{j}}\right), \quad \text { by }(2.9), \\
& <2 \sum_{i=j+1}^{\infty} \frac{1}{\alpha^{i-j}-1}+\sum_{i=j+1}^{\infty} \frac{1}{m_{j}\left(\alpha^{i-j}-1\right)}, \quad \text { by }(2.8), \\
& <2 \sum_{i=1}^{\infty} \frac{1}{\alpha^{i}-1}+\frac{1}{m_{1}}\left(\sum_{i=1}^{\infty} \frac{1}{\alpha^{i}-1}\right), \quad \text { and } \\
t_{j}= & 2 \sum_{i=1}^{j-1} \frac{1}{m_{j} / m_{i}-1}+\sum_{i=1}^{j-1} \frac{1}{m_{i}\left(\alpha^{j-i}-1\right)}, \quad \text { by (2.8) and }(2.10), \\
< & 2 \sum_{i=1}^{j-1} \frac{1}{\alpha^{j-i}-1}+\sum_{i=1}^{j-1} \frac{1}{m_{i}\left(\alpha^{j-i}-1\right)}, \\
< & 2 \sum_{i=1}^{\infty} \frac{1}{\alpha^{i}-1}+\frac{1}{m_{1}} \sum_{i=1}^{\infty} \frac{1}{\alpha^{i}-1} \cdot
\end{aligned}
$$

These inequalities for $s_{j, n}$ and $t_{j}$ are sufficient to deduce that (2.7) holds if for $C$ we take

$$
C=e^{2\left(2+1 / m_{1}\right) \sum_{i=1}^{\infty} 1 /\left(\alpha^{i}-1\right)} .
$$

If $m_{1}=0$ a similar argument suffices to deduce the conclusion.

THEOREM 2.3. Let $S$ be lacunary, consist of integers and for $z_{1}, z_{2} \in \boldsymbol{D}$ let

$$
K\left(z_{1}, z_{2}\right)=\sum_{n=1}^{\infty} p_{n}\left(z_{1}\right) p_{n}\left(z_{2}\right) .
$$

Then the series in (2.11) converges absolutely and uniformly on compact subsets of $\boldsymbol{D} \times \boldsymbol{D}$. Also $A(S)$ is a Hilbert space of analytic functions on $[0,1)$ which has a reproducing kernel given by the restriction of $K$ to $[0,1) \times[0,1)$.

Proof. Let $0 \leqq \delta<1$ and consider $p_{n}(z)$ where $z \in \boldsymbol{D}$ and $|z|<\delta$. Let $C$ be chosen so that (2.7) holds and use (2.1), (2.3), (2.4), and (2.5) to obtain

$$
\begin{aligned}
\left|p_{n}(z)\right| & \leqq C \sqrt{2 m_{n}+1}\left(\sum_{j=1}^{n-1} \frac{2 m_{j}+1}{m_{n}-m_{j}} \delta^{m_{j}}+\delta^{m_{n}}\right), \\
& \leqq C \frac{\sqrt{2 m_{n}+1}}{m_{n}}\left(\sum_{j=1}^{n-1} \frac{2 m_{j}+1}{1-m_{j} / m_{n}} \delta^{m_{j}}+m_{n} \delta^{m_{n}}\right),
\end{aligned}
$$




$$
\begin{aligned}
& <C \frac{\sqrt{2+\frac{1}{m_{n}}}}{\sqrt{m_{n}}}\left(\sum_{j=1}^{n-1} \frac{2 m_{j}+1}{1-\alpha^{j-n}} \delta^{m_{j}}+m_{n} \delta^{m_{n}}\right), \\
& <\frac{C_{0}}{\sqrt{m_{n}}}\left(\sum_{j=1}^{\infty}\left(2 m_{j}+1\right) \delta^{m_{j}}\right), \\
& \quad \text { for some } C_{0} \text {, since } \lim _{n \rightarrow \infty} m_{n}=\infty .
\end{aligned}
$$

Hence we have for all $k$,

$$
\sum_{n=k}^{\infty}\left|p_{n}(z)\right|^{2} \leqq C_{0}^{2}\left(\sum_{j=1}^{\infty}\left(2 m_{j}+1\right) \delta^{m_{j}}\right)^{2}\left(\sum_{n=k}^{\infty} \frac{1}{m_{n}}\right)<\infty .
$$

If we now use (2.12), which is true for all $|z|<\delta$, an application of Schwartz's inequality proves that the series in (2.11) has the stated convergence properties. We also see that if $\sum_{n=1}^{\infty}\left|\alpha_{n}\right|^{2}<\infty$ and if we let $F(z)=\sum_{n=1}^{\infty} \alpha_{n} p_{n}(z)$ for $|z|<1$, then this series converges absolutely and uniformly on compact subsets of $\boldsymbol{D}$ so that $F$ is analytic in $\boldsymbol{D}$. From this we see that $A(S)$ consists of analytic functions on $[0,1)$ and that if $0 \leqq x<1$ and $f \in A(S), f(x)=\sum_{n=1}^{\infty} \alpha_{n} p_{n}(x)$, where $\alpha_{n}$ is the $n$th Fourier coefficient of $f$ with respect to the orthonormal system $\left(p_{n}\right)$. We now see that $K$ is a reproducing kernel for $A(S)$, for (2.12) shows that if $z \in D$ then $x \rightarrow K(z, x)$ belongs to $A(S)$, and if $f=\sum_{n=1}^{\infty} \alpha_{n} p_{n} \in A(S)$, where $\sum_{n=1}^{\infty}\left|\alpha_{n}\right|^{2}<\infty$, then

$$
\begin{aligned}
\int_{0}^{1} K(z, x) f(x) d x & =\lim _{n \rightarrow \infty} \int_{0}^{1}\left(\sum_{k=1}^{n} p_{k}(z) p_{k}(x)\right)\left(\sum_{k=1}^{n} \alpha_{k} p_{k}(x)\right) d x \\
& =\lim _{n \rightarrow \infty} \sum_{k=1}^{n} \alpha_{k} p_{k}(z), \quad \text { by }(2.2), \\
& =f(z), \quad \text { if } \quad z \in[0,1) .
\end{aligned}
$$

REMARK. It should be noted that if $m_{1}=0$ and $\sum_{n=2}^{\infty} 1 / m_{n}=\infty$, then $A(S)$ does not have a reproducing kernel. This is because of the Müntz-Szasz theorem, which shows that in this case $A(S)$ will contain the restrictions to $[0,1)$ of all functions in $C([0,1])$. Hence, if $x \in[0,1)$, the linear functional on $A(S)$ given by $f \rightarrow f(x)$ is not bounded, so that $A(S)$ does not have a reproducing kernel ([2], p. 317).

3. Moment sequences. Let $\left(\alpha_{n}\right)_{n=1}^{\infty}$ be a sequence of complex numbers and let $f \in L^{2}([0,1))$. Consider the condition

$$
\alpha_{n}=\int_{0}^{1} x^{m_{n}} f(x) d x, \quad \text { for } \quad n=1,2,3, \cdots .
$$

THEOREM 3.1. Let $\left(\alpha_{n}\right)_{n=1}^{\infty}$ be a given sequence of complex numbers. Then if $S$ is lacunary, a sufficient condition for (3.1) to hold for some $f$ in $L^{2}$ is that 


$$
\sum_{n=1}^{\infty} m_{n}\left|\alpha_{n}\right|<\infty
$$

This condition is not necessary.

If $S$ satisfies the condition

$$
\sum_{n=2}^{\infty} \frac{\left(\sum_{j=1}^{n-1} m_{j}\right)}{m_{n}}<\infty,
$$

then a sufficient condition for (3.1) to hold for some $f \in L^{2}$ is that

$$
\sum_{n=1}^{\infty} m_{n}\left|\alpha_{n}\right|^{2}<\infty \text {. }
$$

If $S$ satisfies the condition

$$
\sum_{n=2}^{\infty} \frac{\left(\sum_{j=1}^{n-1} \sqrt{m_{j}}\right)^{2}}{m_{n}}<\infty,
$$

then (3.4) is a necessary and sufficient condition for (3.1) to hold for some $f \in L^{2}$.

Proof. By virtue of [2], pp. 226-227, (3.1) holds for some $f \in L^{2}$ if and only if

$$
\sum_{n=1}^{\infty}\left|\sum_{j=1}^{n} \alpha_{n j} \alpha_{j}\right|^{2}<\infty,
$$

in which case $\sum_{n=1}^{\infty}\left(\sum_{j=1}^{n} a_{n j} \alpha_{j}\right) p_{n}$ will do for $f$.

If $S$ satisfies (3.3) or (3.5) then $S$ is lacunary so in any case we may choose $\alpha>1$ so that (2.8) holds when $i>j$. Also, choose $C$ so that (2.7) holds and use (2.3), (2.4), and (2.5) to obtain for $n \geqq 2$,

$$
\left|\sum_{j=1}^{n} a_{n j} \alpha_{j}\right| \leqq C \sqrt{2 m_{n}+1}\left(\left|\alpha_{n}\right|+\sum_{j=1}^{n-1} \frac{2 m_{j}+1}{m_{n}-m_{j}}\left|\alpha_{j}\right|\right),
$$

so that

$$
\left|\sum_{j=1}^{n} a_{n j} \alpha_{j}\right| \leqq \frac{C \sqrt{2 m_{n}+1}}{m_{n}}\left(m_{n}\left|\alpha_{n}\right|+\frac{\alpha}{\alpha-1} \sum_{j=1}^{n-1}\left(2 m_{j}+1\right)\left|\alpha_{j}\right|\right) .
$$

If (3.2) holds this shows that there is $F$ so that

$$
\left|\sum_{j=1}^{n} a_{n j} \alpha_{j}\right| \leqq \frac{F}{\sqrt{m_{n}}}
$$

for all $n \geqq 2$, so that (3.6) and hence (3.1) hold. (3.2) is not necessary since 


$$
1 /\left(m_{n}+1\right)=\int_{0}^{1} x^{m_{n}} d x, \quad \text { but } \sum_{n=1}^{\infty} m_{n} /\left(m_{n}+1\right)=\infty .
$$

Now assume that (3.3) holds. Because of (2.5) and (2.7), (3.4) is equivalent to the condition

$$
\sum_{n=1}^{\infty}\left|a_{n n} \alpha_{n}\right|^{2}<\infty
$$

Also the approach used to derive (3.7) shows that there is a constant $G$, depending only on $S$, so that

$$
\left|\sum_{j=1}^{n-1} \alpha_{n j} \alpha_{j}\right| \leqq \frac{G}{\sqrt{m_{n}}}\left(\sum_{j=1}^{n-1}\left(2 m_{j}+1\right)\left|\alpha_{j}\right|\right),
$$

for $n \geqq 2$.

Now let (3.4) hold. An application of Schwartz's inequality shows that

$$
\left|\sum_{j=1}^{n-1} m_{j} \alpha_{j}\right|^{2} \leqq\left(\sum_{j=1}^{n-1} m_{j}\right)\left(\sum_{j=1}^{n-1} m_{j}\left|\alpha_{j}\right|^{2}\right) \leqq\left(\sum_{j=1}^{n-1} m_{j}\right)\left(\sum_{j=1}^{\infty} m_{j}\left|\alpha_{j}\right|^{2}\right) .
$$

Since $S$ is lacunary and (3.3) holds we have, for some $J, \sum_{n=2}^{\infty}\left|\alpha_{n}\right| \leqq$ $J \sum_{n=2}^{\infty} 1 / \sqrt{m_{j}}<\infty$. These facts, together with (3.3), (3.8), and (3.9) imply that (3.6), and hence (3.1), hold (the latter for some $f \in L^{2}$ ).

Condition (3.5) is stronger than (3.3), so that if (3.5) holds then (3.4) implies (3.1). Conversely let (3.5) and (3.1) hold, the latter for some $f \in L^{2}$. An application of the Schwartz inequality to (3.1) shows that there is a constant $H$ so that $\left|\alpha_{n}\right| \leqq H / \sqrt{m_{n}}$, for $n=$ $2,3, \cdots$. Hence $\sum_{n=1}^{\infty}\left|\alpha_{n}\right|<\infty$ and also

$$
\sum_{j=1}^{n-1} m_{j}\left|\alpha_{j}\right| \leqq H\left(\sum_{j=1}^{n-1} \sqrt{m_{j}}\right) \text {. }
$$

Because of (3.5) we conclude from (3.9) that $\sum_{n=1}^{\infty}\left|\sum_{j=1}^{n-1} a_{n j} \alpha_{j}\right|^{2}<\infty$. Since (3.1) implies (3.6) we deduce that (3.8) holds and this is equivalent to (3.4).

This result suggests introducing a sequence space $L_{S}$ as follows. A sequence $\left(\alpha_{n}\right)_{n=1}$ belongs to $L_{S}$ if and only if there is $f \in L^{2}$ such that $\alpha_{n}=\int_{0}^{1} x^{m} f(x) d x$, for $n=1,2, \ldots$. It is shown in [5], p. 237 that if $S$ consists of integers, then $L_{S} \subseteq \ell^{2}$.

CoRollary 3.2. If $S$ satisfies (3.5), there is a subsequence $S_{1}$ of $S$ so that $L_{S_{1}} \varsubsetneqq L_{S}$. We also have $L_{S} \neq \ell^{2}$, regardless of whether or not $S$ satisfies (3.5).

COROLLARY 3.3. Let $S$ be a lacunary sequence of integers and let $\alpha=\left(\alpha_{n}\right)_{n=1}^{\infty}$ be a given sequence of complex numbers. Then if 
there are real $\gamma$ and $\delta$, with $\delta<1$, so that $\left|\alpha_{n}\right| \leqq \gamma \delta^{m_{n}}$ for $n=1,2, \cdots$, then $\alpha \in L_{S}$.

These results suggest that rather than using the functions $x^{m} n(n=1,2, \cdots)$ in (3.1) it may be more appropriate to use the functions $m_{n} x^{m_{n}}$ or $\sqrt{m_{n}} x^{m_{n}}$. The following is essentially a rewording of Theorem 3.1.

THEOREM 3.4. Let $\left(\alpha_{n}\right)_{n=1}^{\infty}$ be a sequence of complex numbers. If $S$ is lacunary and $\sum_{n=1}^{\infty}\left|\alpha_{n}\right|<\infty$ then there is $f \in L^{2}$ so that $\alpha_{n}=$ $m_{n} \int_{0}^{1} x^{m_{n}} f(x) d x$ for $n=1,2, \cdots$.

If $S$ satisfies (3.5) then $\sum_{n=1}^{\infty}\left|\alpha_{n}\right|^{2}<\infty$ if and only if there is $f \in L^{2}$ so that $\alpha_{n}=\sqrt{m_{n}} \int_{0}^{1} x^{m_{n}} f(x) d x$, for $n=1,2, \cdots$.

REMARKS. If $\mu$ is a measure supported by $[0, \delta]$, where $\delta<1$, and we let $\alpha_{n}=\int_{0}^{1} x^{m_{n}} d \mu(x)$ for $n=1,2, \cdots$ then, assuming that $S$ is a lacunary sequence of integers, Corollary 3.3 applies to give a function $f \in L^{2}$ so that (3.1) holds. That is, the measure $\mu$ can be absolutely continuous with respect to Lebesgue measure.

As remarked in the introduction, if $\sum_{i=2}^{\infty} 1 / m_{i}<\infty$ and $j$ is given, there is a measure $\mu$ on $[0,1]$ so that $\int_{0}^{1} x^{m_{n}} d \mu(x)=0$ if $n \neq j$ and $\int_{0}^{1} x^{m_{j}} d \mu(x) \neq 0$. If $\delta$ is given $(1 \geqq \delta>0)$ and $S$ is a lacunary sequence of integers, the measure $\mu$ can be chosen to be supported by $[0, \delta]$ and be absolutely continuous with respect to Lebesgue measure. To see this, apply Corollary 3.3 to the sequence $\left(\varepsilon_{n} \delta^{m_{n}}\right)_{n=1}^{\infty}$, where $\varepsilon_{n}=0$ if $\mathrm{n} \neq j$ and $\varepsilon_{j}=1$. We obtain $f \in L^{2}$ so that $\varepsilon_{n} \delta^{2 m_{n}}=\int_{0}^{1}(\delta x)^{m_{n}} f(x) d x$, from which the result follows by a change of variable.

\section{REFERENCES}

1. J. A. Clarkson and P. Erdös, Approximation by polynomials, Duke Math. J., 10 (1943), 5-11.

2. P. Davis, Interpolation and Approximation, Blaisdell, 1965.

3. R. E. Edwards, Fourier Series: A Modern Introduction, vols. I and II, Holt, Rinehart and Winston, 1967.

4. C. Goffman and G. Predrick, First Course in Functional Analysis, Prentice Hall, 1965.

5. G. Hardy, J. Littlewood, and G. Pólya, Inequalities, Cambridge, 1967.

6. G. G. Jonson, Moment sequences in Hilbert space, Pacific J. Math., 46 (1973), 201-207.

7. Y. Katznelson, An Introduction to Abstract Harmonic Analysis, Wiley, 1968.

8. W. Rudin, Real and Complex Analysis, McGraw-Hill, 1966.

Received March 18, 1977 and in revised form June 15, 1977.

The University of Wollongong

New South Wales, Australia 


\section{PACIFIC JOURNAL OF MATHEMATICS}

\section{EDITORS}

RICHARD ARENS (Managing Editor)

University of California

Los Angeles, California 90024

C. W. Curtis

University of Oregon

Eugene, OR 97403

C. C. MOORE

University of California

Berkeley, CA 94720

\section{J. DUGUNDJI}

Department of Mathematics University of Southern California Los Angeles, California 90007

R. Finn AND J. Milgram Stanford University Stanford, California 94305

ASSOCIATE EDITORS
E. F. BECKENBACH

B. H. NeUMANN

F. WOLF

K. YoshidA

\section{SUPPORTING INSTITUTIONS}

UNIVERSITY OF BRITISH COLUMBIA UNIVERSITY OF SOUTHERN CALIFORNIA CALIFORNIA INSTITUTE OF TECHNOLOGY STANFORD UNIVERSITY UNIVERSITY OF CALIFORNIA MONTANA STATE UNIVERSITY UNIVERSITY OF TOKYO UNIVERSITY OF NEVADA, RENO UNIVERSITY OF UTAH NEW MEXICO STATE UNIVERSITY OREGON STATE UNIVERSITY UNIVERSITY OF OREGON

WASHINGTON STATE UNIVERSITY UNIVERSITY OF WASHINGTON OSAKA UNIVERSITY 


\section{Pacific Journal of Mathematics}

\section{Vol. 74, No. $1 \quad$ May, 1978}

Gerald Arthur Anderson, Computation of the surgery obstruction groups

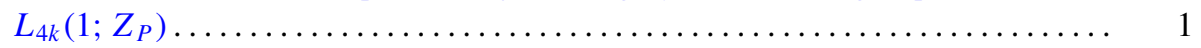

R. K. Beatson, The degree of monotone approximation ................ 5

Sterling K. Berberian, The character space of the algebra of regulated functions . . . 15

Douglas Michael Campbell and Jack Wayne Lamoreaux, Continua in the plane with

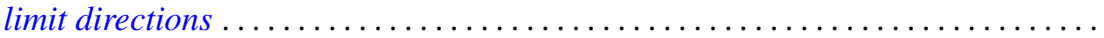

R. J. Duffin, Algorithms for localizing roots of a polynomial and the Pisot

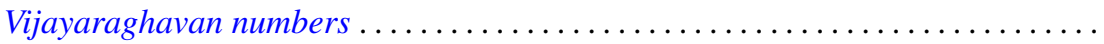

Alessandro Figà-Talamanca and Massimo A. Picardello, Functions that operate on

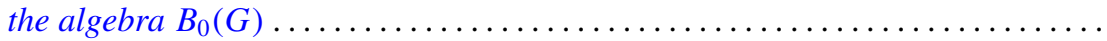

John Erik Fornaess, Biholomorphic mappings between weakly pseudoconvex

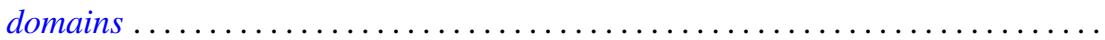

Andrzej Granas, Ronald Bernard Guenther and John Walter Lee, On a theorem of S.

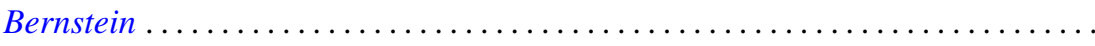

Jerry Grossman, On groups with specified lower central series quotients . .........

William H. Julian, Ray Mines, III and Fred Richman, Algebraic numbers, a constructive development . . . . . . . . . . . . . . . . . . . . . . .

Surjit Singh Khurana, A note on Radon-Nikodým theorem for finitely additive

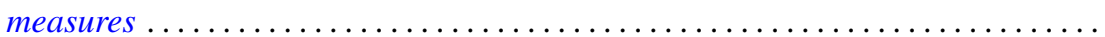

Garo K. Kiremidjian, A Nash-Moser-type implicit function theorem and nonlinear

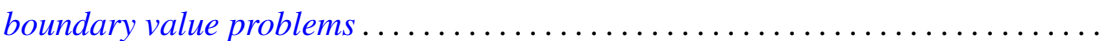

Ronald Jacob Leach, Coefficient estimates for certain multivalent functions ....

John Alan MacBain, Local and global bifurcation from normal eigenvalues. II . . 133

James A. MacDougall and Lowell G. Sweet, Three dimensional homogeneous algebras...

John Rowlay Martin, Fixed point sets of Peano continua ......

R. Daniel Mauldin, The boundedness of the Cantor-Bendixson order of some analytic sets...

Richard C. Metzler, Uniqueness of extensions of positive linear functions ..

Rodney V. Nillsen, Moment sequences obtained from restricted powers . .

Keiji Nishioka, Transcendental constants over the coefficient fields in differential elliptic function fields...

Gabriel Michael Miller Obi, An algebraic closed graph theorem

Richard Cranston Randell, Quotients of complete intersections by $\mathbf{C}^{*}$ actions . . 221

Bruce Reznick, Banach spaces which satisfy linear identities . .

Bennett Setzer, Elliptic curves over complex quadratic fields...

Arne Stray, A scheme for approximating bounded analytic functions on certain subsets of the unit disc.

Nicholas Th. Varopoulos, A remark on functions of bounded mean oscillation and bounded harmonic functions. Addendum to: "BMO functions and the

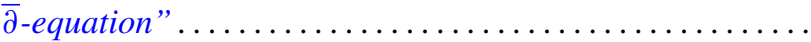

Charles Irvin Vinsonhaler, Torsion free abelian groups quasi-projective over their

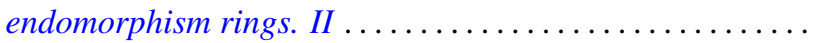

Thomas R. Wolf, Characters of $p^{\prime}$-degree in solvable groups ... 